\title{
Nontrivial solutions of second-order difference equations
}

Yuhua Li

"Correspondence: yhli@sxu.edu.cn School of Mathematical Sciences, Shanxi University, Taiyuan, 030006, People's Republic of China

\begin{abstract}
In this paper, we investigate the nontrivial solutions to the difference equations $-\Delta^{2} u(k-1)=f(k, u(k))$ for all $k \in \mathbb{Z}[0, T]$ and subject to $u(0)=0=\Delta u(T)$, and establish the existence results of a nontrivial solution under some resonant conditions.
\end{abstract}

Keywords: discrete boundary value problem; resonance; critical group

\section{Introduction}

In this paper, we consider the nontrivial solutions to the following discrete boundary value problem:

$$
\left\{\begin{array}{l}
-\Delta^{2} u(k-1)=f(k, u(k)), \quad k \in \mathbb{Z}[1, T], \\
u(0)=0=\Delta u(T),
\end{array}\right.
$$

where $T$ is a positive integer, $\mathbb{Z}[1, T]=\{1,2, \ldots, T\}, \Delta u(k)=u(k+1)-u(k)$.

The discrete boundary value problems have been studied by many authors. Some existence results were obtained by using various methods [1-9]. For example, using the method of upper and lower solutions and the cone expansion or the compression fixed point theorem, authors $[2,3]$ obtained the unique solution and positive solutions to the periodic difference equations. In [6], Jiang and Zhou obtained the existence of nontrivial solutions to BVP (1.1) via the strongly monotone operator principle and the critical point theory. By the mountain pass theorem, multiple positive solutions theorems were established in [1] for discrete problems. However, they did not consider the case that $f$ is resonant at 0 and superlinear at $\infty$. In this paper, we extend the results of [6] to the case that $f$ is resonant at 0 .

Let $\lambda_{1}<\lambda_{2}<\cdots<\lambda_{T}$ denote the eigenvalues of the linear problem corresponding to (1.1). By [6], $\lambda_{i}=4 \sin ^{2} \frac{(2 i-1) \pi}{4 T+2}, i \in \mathbb{Z}[1, T]$, and the corresponding orthogonal eigenvectors of $\left\{\lambda_{i}\right\}_{i=1}^{T}$ are

$$
e_{i}(k)=\sin \frac{2 i-1}{2 T+1} k \pi, \quad k \in \mathbb{Z}[0, T+1], \forall i \in \mathbb{Z}[1, T] .
$$

Assume that the following conditions hold:

$\left(\mathrm{H}_{1}\right) \quad f: \mathbb{Z}[1, T] \times \mathbb{R} \rightarrow \mathbb{R}$ is continuous and $f$ is continuous differentiable on the second variable;

○2013 Li; licensee Springer. This is an Open Access article distributed under the terms of the Creative Commons Attribution License (http://creativecommons.org/licenses/by/2.0), which permits unrestricted use, distribution, and reproduction in any medium, provided the original work is properly cited. 
$\left(\mathrm{H}_{2}\right)$ there exist $m \in \mathbb{Z}[1, T-1]$ and $\delta>0$ such that

$$
\frac{1}{2} \lambda_{m} t^{2} \leq F(k, t) \leq \frac{1}{2} \lambda_{m+1} t^{2}, \quad|t| \leq \delta, k \in \mathbb{Z}[1, T]
$$

where $F(k, t)=\int_{0}^{t} f(k, s) d s$ for all $t \in \mathbb{R}$ and $k \in \mathbb{Z}[1, T] ;$

$\left(\mathrm{H}_{2}^{\prime}\right)$ there exists $\delta>0$ such that

$$
F(k, t) \leq \frac{1}{2} \lambda_{1} t^{2}, \quad|t| \leq \delta, k \in \mathbb{Z}[1, T]
$$

$\left(\mathrm{H}_{3}\right)$ there exist $R_{0}>0$ and $\mu \in(0,1 / 2)$ such that

$$
0<F(k, t) \leq \mu t f(k, t), \quad|t| \geq R_{0}, k \in \mathbb{Z}[1, T]
$$

The following theorems are our main results.

Theorem 1.1 Let $f$ satisfy conditions $\left(\mathrm{H}_{1}\right),\left(\mathrm{H}_{2}\right)$ and $\left(\mathrm{H}_{3}\right)$. Then problem (1.1) has at least a nontrivial solution.

Theorem 1.2 Let $f$ satisfy conditions $\left(\mathrm{H}_{1}\right),\left(\mathrm{H}_{2}^{\prime}\right)$ and $\left(\mathrm{H}_{3}\right)$. Then problem (1.1) has at least a nontrivial solution.

In [6], the authors assumed the following conditions:

( $\left.\mathrm{A}_{1}\right)$ there exist $R_{0}>0$ and $\mu \in(0,1 / 2)$ such that

$$
F(k, t) \leq \mu t f(k, t), \quad|t| \geq R_{0}, k \in \mathbb{Z}[1, T]
$$

$\left(\mathrm{A}_{2}\right) \max _{k \in \mathbb{Z}[1, T]} \lim \sup _{t \rightarrow 0} f(k, t) / t<\lambda_{1}$;

$\left(\mathrm{A}_{3}\right) \min _{k \in \mathbb{Z}[1, T]} \liminf \operatorname{in}_{t \rightarrow+\infty} f(k, t) / t>\lambda_{1}$.

Under conditions $\left(A_{1}\right)-\left(A_{3}\right)$, they obtained that BVP (1.1) has a nontrivial solution. Under $\left(\mathrm{H}_{3}\right)$ and $\left(\mathrm{A}_{2}\right)$, they obtained that BVP $(1.1)$ has a nontrivial solution.

In this paper, we weaken condition $\left(\mathrm{A}_{2}\right)$ to $\left(\mathrm{H}_{2}^{\prime}\right)$ and obtain the same result. Moreover, in condition $\left(\mathrm{A}_{2}\right), f$ does not cross the first eigenvalue. We extend to the case that $f$ may cross $\lambda_{m}$ and $f$ is resonant at 0 to $\lambda_{m}$. Here, $m$ can equal 1 . We also compute the critical groups at $\infty$ and show that the critical groups in a finite dimensional space are different from those in an infinite dimensional space. For an infinite dimensional space, see $[10,11]$.

\section{Lemmas and preliminaries}

In this section, we recall some definitions and lemmas.

Let $H=\{u: \mathbb{Z}[1, T] \rightarrow \mathbb{R}\}$. Then $H$ is a $T$-dimensional Hilbert space with the inner product $(u, v)=\sum_{k=1}^{T} u(k) v(k)$ for all $u, v \in H$, and the corresponding norm $\|u\|=$ $\left(\sum_{k=1}^{T}(u(k))^{2}\right)^{1 / 2}$ for all $u \in H$.

It is well known that the solution of BVP (1.1) is equivalent to the solution of the operator equation

$$
K \mathbf{f} u=u,
$$


where

$$
K u(k)=\sum_{i=1}^{T} G(k, i) u(i), \quad k \in \mathbb{Z}[1, T], \forall u \in H,
$$

$G: \mathbb{Z}[1, T] \times \mathbb{Z}[1, T] \rightarrow \mathbb{Z}[1, T]$ is the Green function for

$$
\left\{\begin{array}{l}
-\Delta^{2} u(k-1)=0, \quad k \in \mathbb{Z}[1, T], \\
u(0)=0=\Delta u(T)
\end{array}\right.
$$

defined by

$$
\begin{array}{ll}
G(k, i)=\min \{k, i\}, & k, i \in \mathbb{Z}[1, T], \\
\mathbf{f} u(k)=f(k, u(k)), & k \in \mathbb{Z}[1, T], \forall u \in H .
\end{array}
$$

In fact, if $u \in H$ is a solution of (2.1), then $v: \mathbb{Z}[0, T+1] \rightarrow \mathbb{R}$ is a solution of BVP (1.1), where

$$
v(k)= \begin{cases}0, & k=0, \\ u(k), & k \in \mathbb{Z}[1, T], \\ u(T), & k=T+1 .\end{cases}
$$

Conversely, if $v$ is a solution of BVP (1.1), then $u$ is a solution to (2.1), where $u(k)=v(k)$ for all $k \in \mathbb{Z}[1, T]$. Thus, we need only to find the solutions to (2.1) in $H$.

In [12-15], by using the property of $K^{1 / 2}$, we convert the solutions to differential equations into the critical points of some functionals. Similarly, the next conclusions hold for BVP (1.1)

\section{Remark 2.1}

(i) By Lemmas 2.1-2.4 in [6], we have that $K: H \rightarrow H$ is a linear continuous, positive definite and symmetric operator. The eigenvalues of $K$ are $\left\{1 / \lambda_{i}\right\}$, where $\lambda_{i}=4 \sin ^{2} \frac{(2 i-1) \pi}{4 T+2}$ for all $i \in \mathbb{Z}[1, T]$, and the corresponding orthogonal eigenvectors of $\left\{1 / \lambda_{i}\right\}_{i=1}^{T}$ are

$$
e_{i}(k)=\sin \frac{2 i-1}{2 T+1} k \pi, \quad k \in \mathbb{Z}[1, T], \forall i \in \mathbb{Z}[1, T] .
$$

Moreover, each eigenvalue $1 / \lambda_{i}$ has algebraic multiplicity 1 for all $i \in \mathbb{Z}[1, T]$.

(ii) $\mathbf{f}: H \rightarrow H$ is bounded and continuous.

(iii) The operator equation (2.1) has a nontrivial solution in $H$ if and only if the operator equation

$$
v=K^{1 / 2} \mathbf{f} K^{1 / 2} v
$$


has a nontrivial solution in $H$. The solution of the operator equation (2.2) in $H$ is equivalent to the critical point of the functional

$$
J(u)=\frac{1}{2}\|u\|^{2}-\sum_{k=1}^{T} F\left(k, K^{1 / 2} u(k)\right),
$$

where $F(k, t)=\int_{0}^{t} f(k, s) d s$ for all $(k, t) \in \mathbb{Z}[1, T] \times \mathbb{R}$, and $J^{\prime}=I-K^{1 / 2} \mathbf{f} K^{1 / 2}$.

In the following, we list some lemmas and preliminaries about the critical group.

Let $E$ be a real Banach space, $f \in C^{1}(E, \mathbb{R}), K=\left\{u \in E: f^{\prime}(u)=0\right\}$ and $f_{c}=\{u \in E$ : $f(u) \leq c\}$.

Definition 2.1 $[10,16]$ Let $p$ be an isolated critical point of $f$, and let $c=f(p)$. We call

$$
C_{q}(f, p)=H_{q}\left(f_{c} \cap U_{p},\left(f_{c} \backslash\{p\}\right) \cap U_{p} ; G\right)
$$

the $q$ th critical group, with the coefficient group $G$ of $f$ at $p$ for all $q \in \mathbb{N}_{0}:=\{0,1,2, \ldots\}$, where $U_{p}$ is a neighborhood of $p$ such that $K \cap\left(f_{c} \cap U_{p}\right)=\{p\}$, and $H_{*}(X, Y ; G)$ stands for the singular relative homology groups with the Abelian coefficient group $G$.

According to the excision property of the singular homology group, the critical groups are well defined; i.e., they do not depend on a special choice of the neighborhood $U_{p}$.

If $f$ satisfies the (PS) condition and the critical values of $f$ are bounded from below on $E$ by some $a \in \mathbb{R}$, then the critical groups of $f$ at infinity

$$
C_{q}(f, \infty)=H_{q}\left(E, f_{a} ; G\right), \quad q \in \mathbb{N}_{0} .
$$

By the deformation lemma, the right-hand side does not depend on the choice of $a$.

The following lemma is the consequence of the Morse inequality, which is sufficient for proving Theorem 1.1.

Lemma 2.1 [17, Proposition 2.1] Suppose that $f \in C^{1}(E, \mathbb{R})$ satisfies the (PS) condition and that $f$ has only finite critical points.

(i) If $C_{k}(f, \infty) \neq 0$ for some $k \in \mathbb{N}_{0}$, then $f$ has a critical point $u$ such that $C_{k}(f, u) \neq 0$.

(ii) Let 0 be an isolated critical point of $f$, and $C_{k}(f, 0) \neq C_{k}(f, \infty)$ for some $k \in \mathbb{N}_{0}$, then $f$ has a nonzero critical point.

Lemma 2.2 Suppose that $\left(\mathrm{H}_{1}\right)$ and $\left(\mathrm{H}_{3}\right)$ hold. Then J satisfies the $(P S)$ condition.

Proof Let $\left\{u_{n}\right\}$ be a sequence along which $\left|J\left(u_{n}\right)\right| \leq C_{1}$ and $J^{\prime}\left(u_{n}\right) \rightarrow 0$, where $C_{1}>0$ is a constant. By conditions $\left(\mathrm{H}_{1}\right)$ and $\left(\mathrm{H}_{3}\right)$, there is a constant $C_{2}>0$ such that

$$
F(k, t) \leq \mu f(k, t) t+C_{2}, \quad(k, t) \in \mathbb{Z}[1, T] \times \mathbb{R} .
$$


It follows from (2.3) that

$$
\begin{aligned}
& J\left(u_{n}\right)-\mu\left(J^{\prime}\left(u_{n}\right), u_{n}\right) \\
& =\left(\frac{1}{2}-\mu\right)\left\|u_{n}\right\|^{2}-\sum_{k=1}^{T}\left[F\left(k, K^{1 / 2} u_{n}(k)\right)-\mu K^{1 / 2} u_{n}(k) f\left(k, K^{1 / 2} u_{n}(k)\right)\right] \\
& \geq(1 / 2-\mu)\left\|u_{n}\right\|^{2}-T C_{2}, \quad n \in \mathbb{N} .
\end{aligned}
$$

Since $\left|J\left(u_{n}\right)\right| \leq C_{1}$ and $\left|\left(J^{\prime}\left(u_{n}\right), u_{n}\right)\right| \leq\left\|u_{n}\right\|$ for $n$ large enough, $\left\{u_{n}\right\}$ is bounded. Thus, $\operatorname{dim} H=T$ implies that $\left\{u_{n}\right\}$ has a convergent subsequence.

In the following, we will compute $C_{q}(J, \infty)$.

Lemma 2.3 Suppose that $\left(\mathrm{H}_{1}\right)$ and $\left(\mathrm{H}_{3}\right)$ hold. Then there is a constant $a_{0}>0$ such that $J_{a} \simeq S$ for all $a<-a_{0}$, where $S$ is the unit sphere in $H$.

Proof By conditions $\left(\mathrm{H}_{1}\right)$ and $\left(\mathrm{H}_{3}\right)$, there exist $C_{3}>0$ and $C_{4}>0$ such that

$$
F(k, t) \geq C_{3}|t|^{1 / \mu}-C_{4}, \quad t \in \mathbb{R}, k \in \mathbb{Z}[1, T]
$$

Thus for any $u \in S$, by (2.3) and (2.4), we have that

$$
\begin{aligned}
J(t u) & =\frac{1}{2} t^{2}-\sum_{k=1}^{T} F\left(k, t K^{1 / 2} u(k)\right) \leq \frac{1}{2} t^{2}-C_{3}|t|^{1 / \mu} \sum_{k=1}^{T}\left|K^{1 / 2} u(k)\right|^{1 / \mu}+C_{4} T \\
& \rightarrow-\infty, \quad t \rightarrow+\infty
\end{aligned}
$$

and

$$
\begin{aligned}
\frac{d}{d t}[J(t u)] & =\left(J^{\prime}(t u), u\right) \\
& =t-\sum_{k=1}^{T} K^{1 / 2} u(k) f\left(k, t K^{1 / 2} u(k)\right) \\
& \leq \frac{2}{t}\left(\frac{1}{2} t^{2}-\frac{1}{2} \sum_{k=1}^{T} t K^{1 / 2} u(k) f\left(k, t K^{1 / 2} u(k)\right)\right) \\
& \leq \frac{2}{t}\left(\frac{1}{2} t^{2}-\frac{1}{2 \mu} \sum_{k=1}^{T} F\left(k, t K^{1 / 2} u(k)\right)+\frac{C_{2} T}{2 \mu}\right) \\
& =\frac{2}{t}\left[\left(\frac{1}{2}-\frac{1}{4 \mu}\right) t^{2}+\frac{1}{2 \mu} J(t u)+\frac{C_{2} T}{2 \mu}\right] \\
& \leq \frac{1}{t \mu}\left[J(t u)+C_{2} T\right], \quad t>0 .
\end{aligned}
$$

Let $a_{0}=C_{2} T+1$. Then, for $a<-a_{0}$, if $J(t u) \leq a$, then $\frac{d}{d t}[J(t u)]<0$. The implicit function theorem is applied to obtain a unique $T \in C(S, \mathbb{R})$ such that

$$
J(T(u) u)=a, \quad u \in S
$$


$T$ has a positive lower bound. In fact, if there is a sequence $\left\{u_{n}\right\} \subset S$ such that $T\left(u_{n}\right) \rightarrow 0$, then $a=J\left(T\left(u_{n}\right) u_{n}\right) \rightarrow J(0)=0$, which is a contradiction. Thus, we may assume that there is $\varepsilon>0$ such that $J(u)>-a$ for all $u \in B_{\varepsilon}$ and $T(u) \geq \varepsilon$ for all $u \in S$, where $B_{\varepsilon}=\{u \in H$ : $\|u\|<\varepsilon\}$. Now, we define a deformation retract $\eta:[0,1] \times\left(H \backslash B_{\varepsilon}\right) \rightarrow\left(H \backslash B_{\varepsilon}\right)$ by

$$
\eta(t, u)= \begin{cases}(1-s) u+s T\left(\frac{u}{\|u\|}\right) \frac{u}{\|u\|}, & u \in\left(H \backslash B_{\varepsilon}\right) \backslash \bigvee_{a}, \\ u, & u \in J_{a} .\end{cases}
$$

Therefore, $H \backslash B_{\varepsilon} \simeq J_{a}$, i.e., $J_{a} \simeq S$.

Lemma 2.4 $[18,19]$ Let 0 be an isolated critical point of $f \in C^{2}(H, \mathbb{R})$ satisfying the (PS) condition, where $H$ is a real Hilbert space. Assume that $f$ has a local linking at 0 with respect to a direct sum decomposition $H=H_{-} \oplus H_{+}$with $k=\operatorname{dim} H_{-}<\infty$, i.e., there exists $r>0$ small such that

$$
f(u)>0, \quad u \in H_{+}, 0<\|u\| \leq r, \quad f(u) \leq 0, \quad u \in H_{-},\|u\| \leq r .
$$

Then $C_{k}(f, 0) \neq 0$.

\section{Proof of main theorems}

In this section, we prove our main theorems.

Proof of Theorem 1.1 Let $H=\{u: \mathbb{Z}[1, T] \rightarrow \mathbb{R}\}$ be the real Hilbert space defined at the beginning in Section 2.

By Lemma 2.2, $J$ satisfies the (PS) condition. We assume that $J$ has only finite critical points. Since $\operatorname{dim} H=T$, it follows from Lemma 2.3 that

$$
C_{q}(J, \infty)=H_{q}\left(H, J_{a}\right) \cong H_{q}\left(D^{T}, S^{T-1}\right)=\delta_{q T} G, \quad q \in \mathbb{N}_{0} .
$$

In the following, we consider the critical groups of $J$ at 0 . Let $H=Y \oplus Z$, where $Y=$ $\operatorname{span}\left\{e_{1}, e_{2}, \ldots, e_{m}\right\}, Z=\operatorname{span}\left\{e_{m+1}, e_{m+2}, \ldots, e_{T}\right\}$. Choosing $r>0$ such that if $\|u\| \leq r$, then $\left|K^{1 / 2} u(k)\right| \leq \delta$ for all $k \in \mathbb{Z}[1, T]$. By condition $\left(\mathrm{H}_{2}\right)$, for $u \in Y$ with $\|u\| \leq r$, we have

$$
\begin{aligned}
J(u) & =\frac{1}{2}\|u\|^{2}-\sum_{k=1}^{T} F\left(k, K^{1 / 2} u(k)\right) \\
& \leq \frac{1}{2}\|u\|^{2}-\frac{1}{2} \sum_{k=1}^{T} \lambda_{m}\left(K^{1 / 2} u(k)\right)^{2} \\
& \leq \frac{1}{2}\|u\|^{2}-\frac{\lambda_{m}}{2 \lambda_{m}}\|u\|^{2}=0 .
\end{aligned}
$$

For $u \in Z=\bigoplus_{k=m+1}^{T} \operatorname{span}\left\{e_{k}\right\}$ with $\|u\| \leq r$, if $u \notin \operatorname{span}\left\{e_{m+1}\right\}$, then we have

$$
\begin{aligned}
J(u) & =\frac{1}{2}\|u\|^{2}-\sum_{k=1}^{T} F\left(k, K^{1 / 2} u(k)\right) \\
& \geq \frac{1}{2}\|u\|^{2}-\frac{1}{2} \sum_{k=1}^{T} \lambda_{m+1}\left(K^{1 / 2} u(k)\right)^{2}
\end{aligned}
$$




$$
\begin{aligned}
& >\frac{1}{2}\|u\|^{2}-\frac{\lambda_{m+1}}{2 \lambda_{m+1}}\|u\|^{2} \\
& =0 .
\end{aligned}
$$

If there is $u_{0} \in \operatorname{span}\left\{e_{m+1}\right\}$ with $0<\left\|u_{0}\right\| \leq r$ such that $J\left(u_{0}\right)=0$, then it follows from the above inequality and condition $\left(\mathrm{H}_{2}\right)$ that

$$
F\left(k, K^{1 / 2} u_{0}(k)\right)=\frac{1}{2} \lambda_{m+1}\left(K^{1 / 2} u_{0}(k)\right)^{2}, \quad k \in \mathbb{Z}[1, T]
$$

By (3.1) and Rolle's theorem, there exists $t_{k} \in(0,1)$ such that

$$
f\left(k, t_{k} K^{1 / 2} u_{0}(k)\right)=\lambda_{m+1} t_{k}\left(K^{1 / 2} u_{0}(k)\right), \quad k \in \mathbb{Z}[1, T] .
$$

Let $u(k)=t_{k} u_{0}(k)$ for all $k \in \mathbb{Z}[1, T]$. Then $u$ is also a critical point of $J$ and $u$ is nonzero. The conclusion holds.

If there is not $u \in \operatorname{span}\left\{e_{m+1}\right\}$ with $0<\|u\| \leq r$ such that $J(u)=0$, then

$$
J(u) \leq 0, \quad u \in Y,\|u\| \leq r, \quad J(u)>0, \quad u \in Z, 0<\|u\| \leq r .
$$

Thus, $J$ has a local linking at 0 with respect to the decomposition $H=Y \oplus Z$. Since $\operatorname{dim} Y=$ $m$, by Lemma 2.4 , we deduce that $C_{m}(J, 0) \neq 0$. Since $m \neq T$, by Lemma 2.1 , we know that $J$ has a nonzero critical point $u$. The proof is completed.

Proof of Theorem 1.2 Since condition $\left(\mathrm{H}_{3}\right)$ holds, we have by Lemma 2.3 and the proof of Theorem 1.1 that $C_{q}(J, \infty)=\delta_{q T} G$ for all $q \in \mathbb{N}_{0}$.

In the following, we compute the critical groups of $J$ at 0 . Choosing $r>0$ such that if $\|u\| \leq r$, then $\left|K^{1 / 2} u(k)\right| \leq \delta$ for all $k \in \mathbb{Z}[1, T]$. For $u \in H=\bigoplus_{k=1}^{T} \operatorname{span}\left\{e_{k}\right\}$ with $\|u\| \leq r$, if $u \notin \operatorname{span}\left\{e_{1}\right\}$, then we have

$$
\begin{aligned}
J(u) & =\frac{1}{2}\|u\|^{2}-\sum_{k=1}^{T} F\left(k, K^{1 / 2} u(k)\right) \\
& \geq \frac{1}{2}\|u\|^{2}-\frac{1}{2} \sum_{k=1}^{T} \lambda_{1}\left(K^{1 / 2} u(k)\right)^{2} \\
& >\frac{1}{2}\|u\|^{2}-\frac{\lambda_{1}}{2 \lambda_{1}}\|u\|^{2} \\
& =0 .
\end{aligned}
$$

If there is $u_{0} \in \operatorname{span}\left\{e_{1}\right\}$ with $0<\left\|u_{0}\right\| \leq r$ such that $J\left(u_{0}\right)=0$, then it follows from the above inequality and condition $\left(\mathrm{H}_{2}^{\prime}\right)$ that

$$
F\left(k, K^{1 / 2} u_{0}(k)\right)=\frac{1}{2} \lambda_{1}\left(K^{1 / 2} u_{0}(k)\right)^{2}, \quad k \in \mathbb{Z}[1, T]
$$

By (3.2) and Rolle's theorem, there exists $t_{k} \in(0,1)$ such that

$$
f\left(k, t_{k} K^{1 / 2} u_{0}(k)\right)=\lambda_{1} t_{k}\left(K^{1 / 2} u_{0}(k)\right), \quad k \in \mathbb{Z}[1, T]
$$


Let $u(k)=t_{k} u_{0}(k)$ for all $k \in \mathbb{Z}[1, T]$. Then $u$ is also a critical point of $J$ and $u$ is nonzero. The conclusion holds.

If there is not $u \in \operatorname{span}\left\{e_{1}\right\}$ with $0<\|u\| \leq r$ such that $J(u)=0$, then

$$
J(u)>0, \quad 0<\|u\| \leq r .
$$

Then 0 is a local minimum of $J$. So, we deduce that

$$
C_{q}(J, 0)=\delta_{q 0} G
$$

Since $0 \neq T$, by Lemma 2.1, we know that $J$ has a nonzero critical point $u$. The proof is completed.

Corollary 3.1 Suppose that $\left(\mathrm{H}_{1}\right),\left(\mathrm{H}_{3}\right)$ hold and that

$$
\limsup _{t \rightarrow 0} f(k, t) / t<\lambda_{1}, \quad k \in \mathbb{Z}[1, T] .
$$

Then BVP (1.1) has at least a nontrivial solution.

Proof By the assumptions, we need only to verify condition $\left(\mathrm{H}_{2}^{\prime}\right)$. In fact, by (3.3), there is $\delta>0$ such that

$$
F(k, t)<\frac{1}{2} \lambda_{1} t^{2}, \quad 0<|t| \leq \delta, k \in \mathbb{Z}[1, T] .
$$

It follows from Theorem 1.2 that the conclusion holds.

Remark 3.1 In condition $\left(\mathrm{H}_{2}^{\prime}\right)$, the $\operatorname{limit}_{t \rightarrow 0} f(k, t) / t$ may equal $\lambda_{1}$, which is a progress of [6]. Moreover, we also extend to $\lambda_{m}$ in condition $\left(\mathrm{H}_{2}\right)$.

Remark 3.2 Since $\operatorname{dim} H<\infty$, it follows from condition $\left(\mathrm{H}_{3}\right)$ that $C_{q}(J, \infty)=\delta_{q T} G$ for all $q \in \mathbb{N}_{0}$. This is different from $\operatorname{dim} H=\infty$, in which $C_{q}(J, \infty)=0$ for all $q \in \mathbb{N}_{0},[10,11]$.

\section{Example 3.1 Let}

$$
f(k, t)=\frac{1}{2}\left(\lambda_{m}+\lambda_{m+1}\right) \sin t+t^{3}, \quad t \in \mathbb{R} .
$$

Then $f$ satisfies all the conditions of Theorem 1.1. We will obtain one nontrivial solution.

\section{Competing interests}

The author declares that he has no competing interests.

Author's contributions

The author worked on the results independently.

\section{Acknowledgements}

The author is very grateful to the referees for their helpful comments. This paper is supported by the National Natural Science Foundation of China (Grant No. 11071149, 11301313) and Science Council of Shanxi Province (2010011001-1, 2012011004-2, 2013021001-4). 


\section{References}

1. Agarwal, RP, Perera, K, O'Regan, D: Multiple positive solutions of singular and nonsingular discrete problems via variational methods. Nonlinear Anal. 58, 69-73 (2004)

2. Atici, FM, Cabada, A: Existence and uniqueness results for discrete second order periodic boundary value problems Comput. Math. Appl. 45, 1417-1427 (2003)

3. Atici, FM, Guseinov, GS: Positive periodic solutions for nonlinear difference equations with periodic coefficients. J. Math. Anal. Appl. 232, 166-182 (1999)

4. Bin, $H, Y u, J, G u o, Z$ : Nontrivial periodic solutions for asymptotically linear resonant difference problem. J. Math. Anal. Appl. 322, 477-488 (2006)

5. He, Z, Yu, J: On the existence of positive solutions of fourth-order difference equations. Appl. Math. Comput. 161, 139-148 (2005)

6. Jiang, L, Zhou, Z: Existence of nontrivial solutions for discrete nonlinear two point boundary value problems. Appl. Math. Comput. 180, 318-329 (2006)

7. Zhu, B, Yu, J: Multiple positive solutions for resonant difference equations. Math. Comput. Model. 49, 1928-1936 (2009)

8. Zhou, Z, Yu, J, Guo, Z: Periodic solutions of higher dimensional discrete system. Proc. R. Soc. Edinb. A 134, 1013-1022 (2004)

9. Zhou, Z, Yu, J, Guo, Z: The existence of periodic and subharmonic solutions to subquadratic discrete Hamiltonian ANZIAM J. 47, 89-102 (2005)

10. Chang, K-C: Infinite Dimensional Morse Theory and Multiple Solution Problems. Birkhäuser, Boston (1993)

11. Han, G, Xu, Z: Multiple solutions of some nonlinear fourth-order beam equations. Nonlinear Anal. 68, 3646-3656 (2008)

12. Li, F, Li, Y, Liang, Z: Existence and multiplicity of solutions to $2 m$ th-order ordinary differential equations. J. Math. Anal. Appl. 331, 958-977 (2007)

13. Li, F, Li, Y, Liang, Z: Existence of solutions to nonlinear Hammerstein integral equations and applications. J. Math. Anal. Appl. 323, 209-227 (2006)

14. Li, F, Liang, Z, Zhang, Q: Existence of solutions to a class of nonlinear second order two-point boundary value problems. J. Math. Anal. Appl. 312, 357-373 (2005)

15. Li, F, Zhang, Q, Liang, Z: Existence and multiplicity of solutions of a kind of fourth-order boundary value problem. Nonlinear Anal. 62, 803-816 (2005)

16. Mawhin, J, Willem, M: Critical Point Theory and Hamiltonian Systems. Springer, Berlin (1989)

17. Liu, S: Nontrivial solutions for elliptic resonant problems. Nonlinear Anal. 70, 1965-1974 (2009)

18. Liu, J: A Morse index for a saddle point. Syst. Sci. Math. Sci. 2, 32-39 (1989)

19. Su, J: Semilinear elliptic boundary value problems with double resonance between two consecutive eigenvalues. Nonlinear Anal. 48, 881-895 (2002)

10.1186/1687-1847-2013-365

Cite this article as: Li: Nontrivial solutions of second-order difference equations. Advances in Difference Equations 2013, 2013:365

\section{Submit your manuscript to a SpringerOpen ${ }^{\circ}$ journal and benefit from:}

- Convenient online submission

- Rigorous peer review

- Immediate publication on acceptance

- Open access: articles freely available online

- High visibility within the field

- Retaining the copyright to your article 